Review

\title{
MODERN COMPLEX NON-INVASIVE METHODS FOR DIAGNOSING ISCHEMIC DISORDERS OF CEREBRAL CIRCULATION
}

\author{
L. Manchev ${ }^{\text {*, T. Manolova }}{ }^{2}$, V. Mancheva-Ganeva², I. Manchev² \\ ${ }^{1}$ Department of Diagnostic Imaging, Faculty of Medicine, Trakia University, Stara Zagora, Bulgaria \\ ${ }^{2}$ Department of Neurology and Psychiatry, Faculty of Medicine, Trakia University, \\ Stara Zagora, Bulgaria
}

\begin{abstract}
Cerebral ischemic strokes (cerebral infarctions) represent $80 \%$ of the acute disorders of cerebral circulation. They most frequently occur in cases of atherosclerosis of the extra- and intracranial vessels and /or arterial hypertension, in cardiac diseases (, rhythm and conduction disorders), cerebral vasculitis, blood diseases, related to hypercoagulability (trombocytosis), etc. One of the welldocumented risk factors for the cerebrovascular disease is the asymptomatic carotid stenoses (ACS) and the symptomatic ones, especially those that have hemodynamic significance.

In a lot of cases the use of CT, MRT of the brain and Doppler sonography is not informative enough for the understanding of the etiopathogenesis of cerebral strokes. This is true especially for those, having occurred in the vertebrobasilar vascular system. This calls for the combined use of several diagnostic methods, especially in the cases in which the genesis of ischemic strokes can be explained with the simultaneous influence of several risk factors.
\end{abstract}

Key words: cerebral ischemic stroke, computed tomography, magnetic-resonance tomography

The neuroimaging techniques most frequently used in diagnosing ischemic disorders of cerebral circulation are: Computed Tomography (CT), Magnetic Resonance Tomography MRT) and Doppler Sonography (DSG).

If there is any suspicion of a cerebral infarction (CI) or a transient ischemic attack (TIA), slow conduction of CT or MRT examination is recommended, together with ultrasound examination of extra- and intracranial circulation (1).

Noncontrast computed tomography of the brain is a widely available method, providing for reliable CI diagnosing and its being distinguished from brain haemorrage in the first few days after occurrence $(2,3)$.

During CT scan early ischemic changes show hypodense zones, perifocal edema and deletion of corticomeddulary border (4).

*Correspondence to: Lachezar Manchev, MD, PhD, Medical Faculty, Trakian University, Stara Zagora, 11, "Armeyska “str., 6003, Stara Zagora, 0887 438703,Email:lachezar_manchev@yahoo.it
Magnetic resonance tomography is more widely used as compared to CT in diagnosing vertebrobasilar system $\mathrm{CI}$, as well as lacunar and cortical ischemic strokes $(5,6,7,8)$.

Doppler sonography examination of extracranial blood vessels is a highly informative method in diagnosing vascular accidents as opposed to those of the vertebrobasilar system, which, in spite of providing good results, are not always precise (9).

Early Doppler sonography examination in cases of acute ischemic stroke allows for timely diagnosis of different stages of carotid stenoses (1). The method is very useful for examining recurrent cerebral strokes as well (10).

Computed Tomography and Magnetic Resonance Tomography

The diagnostic features of Computed Tomography and Magnetic Resonance Tomography were compared in cases of acute cerebral strokes. MRT was proven to be a more sensitive and specific method as compared to non-contrast CT $(11,12,13)$. 
Computed Tomography is a more suitable method for diagnosing comatose patients.

\section{Computed Tomography (CT) and Doppler Sonography (DSG)}

Spiral computer tomography is a comparatively new diagnostic method, which allows non-invasive diagnosis of cerebral strokes, especially in combination with Doppler sonography. A total of 59 patients with clinical suspicion for carotid artery obstruction, found with CT and Doppler Sonography scans, were studied. The Doppler Sonography scan found 4 complete obturations, 38 severe stenoses (80-99\%) and 32 moderate stenoses (30-69\%).In 5 patients, in whom the Duplex scanning did not provide an adequate evaluation of the carotid artery because of a small spectral window or severe calcification, the Spiral CT made it possible to identify and measure correctly the stenotic lesion. The study showed that spiral CT scan of the extracranial cerebral arteries is a promising non-invasive additional method to be used in the cases when Duplex scanning is not reliable enough (kinking, severe calcification, short neck) and particularly when the situation calls for a complete scan of the vascular wall (14).

The incidence, severity and clinical significance of carotid atherosclerosis in patients with ischemic stroke with nonvalvular atrial fibrillations were studied. 103 patients (64 men and 39 women of average age of 69 years) suffering from ischemic stroke with non-valvular atrial fibrillations underwent carotid Duplex sonography and computed tomography scans within 3 to 7 days after the stroke onset. High-grade stenoses $>50 \%$ of the extracranial carotid artery were found in 25 patients $(24,3 \%)$, including 11 patients $(10,6$ $\%)$ with internal carotid artery obstruction. In $15(66,7 \%)$ of the patients with high-grade carotid stenoses the lesions were situated ipsilaterally to the infarction, including 8 cases with internal carotid artery occlusion. Patients with high-grade stenosis used to smoke more cigarettes $(p<0,05)$ and more often suffered from diseases of the peripheral arteries. The clinical manifestations (transient ischemic attacks, lacunar or massive strokes) did not indicate a significant difference in the groups with stenosis $<50 \%$ and $>50 \%$. Patients with severer stenoses died more frequently. Patients with higher grade of carotid stenosis more often had CT scan findings for cortical stroke $(p<0,01)$. Six of seven patients with haemorrhagic transformation of the ischemic stroke, found by the CT scan, had stenoses of lower grade. The survey showed that the ischemic stroke with non-valvular atrial fibrillations is not always cardiogenic. Carotid stenosis of higher grade was found in one fourth of the patients with ischemic stroke and those with non-valvular atrial fibrillations and the condition was more frequently related to cortical cerebral infarction (15).

The diagnostic value of Computed tomography scan and Carotid Doppler sonograhy were compared in 115 patients with asymptomatic carotid stenoses, 203 patients with transient ischemic attacks and carotid stenosis, and 63 patients with transitory ischemic attacks, but without carotid stenoses. The computed tomography scans most frequently found lesions in patients with TIA, combined with carotid stenosis - $(47 \%)$ as compared to the other groups $(30 \%, 19 \%), \mathrm{p}<0,001$. Cerebral stroke, ipsilateral to the carotid stenosis was found in $10 \%$ of the patients with mediumgrade stenoses $(35-50 \%)$. In patients with asymptomatic carotid stenoses (ACS), $68 \%$ of the strokes were ipsilateral to the stenoses. In patients with TIA and symptomatic carotid stenoses, $86 \%$ of the strokes were also ipsilateral to the stenosis.

The study showed that severer the carotid stenosis, the higher the incidence of cerebral stroke, situated ipsilateral to the stenosis. "Silent" cerebral stroke can be regarded as an indication for carotid endarterectomy in asymptomatic patients.

A total of ninety-six patients with carotid stenoses greater than $50 \%$, who had undergone carotid endarterectomy (59 men and 37 women of average age 69,5 years) were examined by bilateral Duplex scanning for measuring the carotid stenosis, and by CT scan for detecting cerebral strokes. The findings showed good correlation with the data from the CT scan for cerebral strokes. Thirtytwo percent of the patients with symptomatic carotid stenosis had positive CT scan result as compared to $16 \%$ of the patients with asymptomatic plaques (16).

Computed Tomography, Doppler Sonography and Magnetic Resonance Tomography (MRT) Comparative studies with Doppler sonography, Computed tomography and MRT were conducted in new-born babies who were clinically suspicious for intracranial ischemia or haemorrhagia, the aim of the studies having been to determine the most suitable technique. Forty-seven of the newborn babies, who were clinically suspicious for hypoxic ischemic injuries, were examined by $\mathrm{CT}$ and/ 
or MRT and Doppler sonography within the first month of their lives. The CT scan and the MRT showed high degree of coincidence regarding the detection of cortical hypoxic ischemic injuries and cerebral haemorrhages as compared to the Doppler Sonography test MRT and CT scans found 25 cases of ischemic injuries as compared to 13 cases, found by Doppler Sonography. They also found 10 cases of intraparenchymal haemorrhages as compared to the Doppler sonography test. The findings show that CT and MRT scans, when used for detection of ischemic and haemorrhagic disorders, have better diagnostic significance in comparison with the Doppler sonography tests (17).

Calcium and phosphorus are the minerals, which are most frequently found in the human body and are included in a wide range of biochemical pathways, participating predominantly in the formation of calcium hydroxylapatite. Although it is the basic component of bones and teeth, it can also be deposited, together with other forms of Calcium Phosphate, in the blood vessels, the deposition leading to adverse consequences. The process, known as vascular calcification, may eventually lead to stenosis of the blood vessels, ischaemia and death. Approximately $90 \%$ of the patients with cardiovascular diseases have vascular calcification. A study was conducted with the purpose of evaluating the informative value of the different methods for quantitative measuring of the calcification in vivo. Autopsy material and material from carotid endarterectomy was studied with the aim of measuring the calcium content. B-mode ultrasonography, intravascular ultrasound, electron beam computed tomography, MRT and histological methods were used. The study makes it possible to evaluate the calcification degree of the atherosclerotic lesions and choose the most appropriate therapeutic approach for their treatment (18).

\section{ABBREVIATIONS \\ ACS Asymptomatic carotid stenoses \\ CI Cerebral Infarction \\ CT Computed Tomography \\ DSG Doppler Sonography \\ MRT Magnetic Resonance Tomography \\ TIA Transient ischemic attack}

\section{REFERENCES}

1. Guidelines for Management of Ischaemic Stroke and Transient Ischaemic Attack, 2008

2. Wardlaw J, Seymor J, Cairns J et al. Immediate computed tomography scanning of acute stroke is cost-effective and
MANCHEV L., et al. improves quality of life. Stroke 2004, 35: 2477

3. Butcher $K$, Lee $S$, Parsons $M$ et al. Differential prognosis of isolated cortical swelling and hypoattenuation of CT in acute stroke. Stroke , 2007, 38:941

4. Von Kummer R, Bourquan H, Bastianello S et al. Early prediction of irrevestible brain damage offer ischemic stroke at CT. Radiology, 2001, 219: 95-100

5. Rosso C,Drier A, Lacroix D et al. Diffusion -weighted MRI in acute stroke within the first 6 hours: 1,5 or 3.0 tesla? Neurology 2010; 74: 1946

6. Schellinger PD, Bryan RN, Caplan LR et al. Evidence-based guideline: The role of diffusion and perfusion MRI for the diagnosis of acute ischemic stroke: report of the report of the Therapeutics and Technology Assessment Subcommittee of the American Academy of Neurology. Neurology 2010; 75: 177

7. Ogata T, Nagakane $Y$, Christensen $S$ et al. A topographic study of the evolution of the MR DWI/PWI mismatch pattern and its clinical impact: a study by the EPITHET and DEFUSE Investigators. Stroke 2011; 42: 1596

8. Gonzalez R.Clinical MR of acute ischemic stroke. J .Magn Reson. Imaging, 2012, 36, 259-271.

9. Naqvi J, Yap K, Ahmad G. Transcranial Doppler Ultrasound: A Review of the Physical Principles and Major Applications in Critical Care. International Journal of Vascular Medicine, 2013

10.Telman G, Hurani H, Sprecher E et al. Middle Cerebral Artery Stenosis in Patients with Acute Ischemic Stroke and TIA in Israel. Brain, 2015, 36, 46-49

11.Davis D, Robertson T, Imbesi S.Diffusionweighted magnetic resonance imaging versus computed tomography in the diagnosis of acute ischemic stroke. J Emerg Med, 2006, 31,269-277

12. Chalela JA, Kidwell CS, Nentwich LM et al. Magnetic resonance imaging and computed tomography in emergency assessment of patients with suspected acute stroke: a prospective comparison. Lancet 2007; 369: 293

13.Brazzelli M, Sandercock PA, Chappell FM et al. Magnetic resonance imaging versus computed tomography for detection of acute vascular lesions in patients presenting with stroke symptoms. Cochrane Database Syst Rev 2009; CD007424

14.Corti R, Ferrari C, Roberti M et al. Spiral Computed Tomography.A Novel Diagnostic Approach for Investigation of 
the Extracranial Cerebral Arteries and its Complementary Role in Duplex. Ciculation, 1998,98: 984-989

15.Chang XJ, Ryu SL, Lin SK.Carotid Artery Stenosis in Ischemic Stroke Patients with Nonvalvular Arterial Fibrilation. Cerebrovasc. Dis.,2002,13: 16-20

16. Biasi GM, Mingazzini Pm, Baronio L et al.Carotid Plaque Characterization Using Digital Image Processing and its Potential in future Studies of Carotid Endarterectomy
MANCHEV L., et al. and Angioplastyc. Journal of Endovasc. therapy, 1990, vol.5 №3 , 240-246

17. Blankenberg FG, Loh NN, Bracci $P$ et al. Sonography CT and MR Imaging: A Prospective Comparison of Neonates with Suspected Intracranial Ischemia and Hemorrhage.American Journal of Neuroradiology, 2000, 21: 213-218.

18.Higgins CL, Marvel SA, Morrisett JD.Quantification of Calcification in Atherosclerotic lesions. Atheroscler Thromb Vasc Biol, 2005,25: 1567-1576 